\title{
Caldera geometry determined by the depth of the magma chamber
}

\author{
Shigekazu Kusumoto ${ }^{1}$ and Keiji Takemura ${ }^{2}$ \\ ${ }^{1}$ School of Marine Science and Technology, Tokai University, 3-20-1 Shimizu-ku, Orido, Shizuoka 424-8610, Japan \\ ${ }^{2}$ Institute for Geothermal Sciences, Graduate School of Science, Kyoto University, Noguchibaru, Beppu, Oita 874-0903, Japan
}

(Received May 20, 2005; Revised September 10, 2005; Accepted September 12, 2005)

\begin{abstract}
The depth of the magma chamber is shown to be an important factor governing the initial type, scale, and collapse of a caldera. The collapse of the magma chamber is approximated by the contraction of a sphere in an elastic medium, and the distribution of plastic and/or rupturing area on the surface is calculated using the Coulomb failure criterion under the assumption of an elastic-perfectly plastic material. It is found that the necessary contraction for the formation of a caldera is described by fifth-power polynomial expression of the depth of the magma chamber, and that the radius and geometry of the caldera are dependent on the depth of the magma chamber.
\end{abstract}

Key words: Caldera, magma chamber, caldera geometry, depth of magma chamber, numerical simulation.

\section{Introduction}

Numerous laboratory experiments and numerical simulations have been conducted in an attempt to explain the mechanism of caldera formation (e.g., Komuro, 1987; Marti et al., 1994; Gudmundsson et al., 1997; Gudmundsson, 1998; Acocella et al., 2000; Roche et al., 2000; Kusumoto and Takemura, 2003). However, none have reached a quantitative discussion of the factors dominating caldera formation. Recent laboratory experiments and field research have shown that the piston- and funnel-type calderas have the same collapse mechanism and/or physical processes, and the size and depth of magma chamber have been suggested to be important factors governing the type of caldera eventually formed (e.g., Roche et al., 2000; Lipman, 1997).

In this study, in order to clarify whether the depth of the magma chamber controls the caldera geometry or not, the relationships between caldera geometry and the depth of the chamber are discussed quantitatively. Although it is known that the rupture would result in dyke injection, the discussion was focused on the collapse of the magma chamber because it is difficult to consider the effects due to the dyke injection in the simulations. And, in order to facilitate mathematical and physical treatment, a spherical model is employed here instead of the ellipsoidal model (sill-like chamber) widely regarded as the common shape of a magma chamber.

The collapse of the magma chamber is approximated by the contraction of a sphere in an elastic medium, and the distribution of plastic and/or rupturing area on the surface is calculated using the Coulomb failure criterion under the assumption of an elastic-perfectly plastic material (Fig. 1). The elastic-plastic boundary is assumed to lie on the structural boundary of the caldera. The effects due to differences

Copy right(c) The Society of Geomagnetism and Earth, Planetary and Space Sciences (SGEPSS); The Seismological Society of Japan; The Volcanological Society of Japan; The Geodetic Society of Japan; The Japanese Society for Planetary Sciences; TERRAPUB. in models (point source model (e.g., Mogi, 1958; Hagiwara, 1978) and finite sphere model (e.g., Tsuchida and Nakahara, 1972; McTigue, 1987)) and analytic dimensions are also evaluated in the simulations. Point source and finite sphere models are compared through two-dimensional analyses.

\section{Models and Simulations}

Approximating the magma chamber as a small sphere (point source) in an elastic medium, the surface strain fields due to radial deformation of the small sphere are obtained from the analytical solutions of three-dimensional deformation (Hagiwara, 1978) as follows.

$$
\begin{aligned}
\varepsilon_{x x} & =\frac{a^{3} \Delta p}{2 \mu R^{3}} \frac{\lambda+2 \mu}{\lambda+\mu}\left(1-\frac{3 x^{2}}{R^{2}}\right), \\
\varepsilon_{y y} & =\frac{a^{3} \Delta p}{2 \mu R^{3}} \frac{\lambda+2 \mu}{\lambda+\mu}\left(1-\frac{3 y^{2}}{R^{2}}\right), \\
\varepsilon_{z z} & =-\frac{a^{3} \Delta p}{2 \mu R^{3}} \frac{\lambda}{\lambda+\mu}\left(1-\frac{3 d^{2}}{R^{2}}\right), \\
\varepsilon_{x y} & =-\frac{3 a^{3} \Delta p}{2 \mu R^{3}} \frac{\lambda+2 \mu}{\lambda+\mu} \frac{x y}{R^{2}} \\
\varepsilon_{y z} & =-\frac{3 a^{3} \Delta p}{2 \mu R^{3}} \frac{\lambda+2 \mu}{\lambda+\mu} \frac{y d}{R^{2}} \\
\varepsilon_{z x} & =-\frac{3 a^{3} \Delta p}{2 \mu R^{3}} \frac{\lambda+2 \mu}{\lambda+\mu} \frac{x d}{R^{2}}
\end{aligned}
$$

where $\lambda$ and $\mu$ are Lame's constants, $R^{2}=x^{2}+y^{2}+d^{2}$, and $\Delta p$ is the internal pressure change of the sphere, which is related to the volume change $(\Delta V)$ by

$$
\Delta p=\frac{\mu \Delta V}{\pi a^{3}} .
$$

By introducing the contraction $(\Delta a)$ of the magma chamber, the internal pressure change $\Delta p$ can also be expressed 


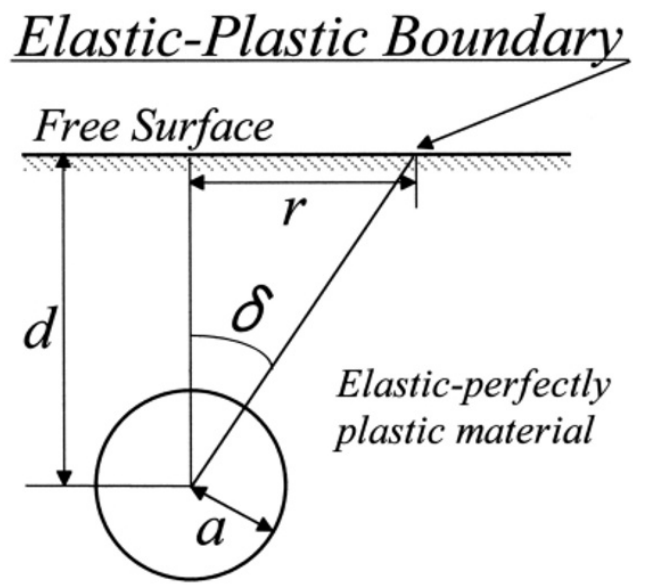

Fig. 1. Model of spherical magma chamber with radius $a$ and depth to centre $d$, where $r=\left(x^{2}+y^{2}\right)^{1 / 2}$ is the horizontal distance from the top of the magma chamber to the plastic area. The crust is assumed to react as an elastic-perfectly plastic material.

as

$$
\Delta p=\frac{4 \mu}{3}\left[\left(\frac{\Delta a}{a}\right)^{3}-3\left(\frac{\Delta a}{a}\right)^{2}+3\left(\frac{\Delta a}{a}\right)\right]
$$

Three-dimensional deformations due to contraction of the small sphere given by Hagiwara (1978) are described as follows.

$$
\begin{aligned}
& u=\frac{a^{3} \Delta p}{4 \mu} r\left[\frac{1}{R_{1}^{3}}+\frac{\lambda+3 \mu}{\lambda+\mu} \frac{1}{R_{2}^{3}}-\frac{6 z(z+d)}{R_{2}^{5}}\right] \\
& w=\frac{a^{3} \Delta p}{4 \mu}\left[\frac{d-z}{R_{1}^{3}}+\frac{2 d}{R_{2}^{3}}-\frac{\lambda-\mu}{\lambda+\mu} \frac{z+d}{R_{2}^{3}}+\frac{6 z(z+d)^{2}}{R_{2}^{5}}\right]
\end{aligned}
$$

where $u$ and $w$ are displacement in the $r$ and $z$ directions. The parameter $R_{1}$ and $R_{2}$ are defined as follows.

$$
R_{1}=\sqrt{r^{2}+(d-z)^{2}}, \quad R_{2}=\sqrt{r^{2}+(d+z)^{2}}
$$

In the case of $z=0$ and $\lambda=\mu$, the Hagiwara's model is equivalent to the Mogi's model (Mogi, 1958).

The surface strain fields due to radial deformation of a finite sphere are obtained from the analytical solutions of two-dimensional deformation (McTigue, 1987) as follows.

$$
\begin{aligned}
\varepsilon_{x x}= & \frac{A}{d^{2}\left(\rho^{2}+1\right)^{5 / 2}} \\
& \cdot\left[d^{2}\left(\rho^{2}+1\right)\left(B+\frac{C}{\rho^{2}+1}\right)-x^{2}\left(3 B+\frac{5 C}{\rho^{2}+1}\right)\right] \\
\varepsilon_{y y}= & \frac{A}{d^{2}\left(\rho^{2}+1\right)^{5 / 2}} \\
& \cdot\left[d^{2}\left(\rho^{2}+1\right)\left(B+\frac{C}{\rho^{2}+1}\right)-y^{2}\left(3 B+\frac{5 C}{\rho^{2}+1}\right)\right] \\
\varepsilon_{x y}= & -\frac{A x y}{d^{2}\left(\rho^{2}+1\right)^{5 / 2}}\left(3 B+\frac{5 C}{\rho^{2}+1}\right)
\end{aligned}
$$

where $\rho=\left(x^{2}+y^{2}\right)^{1 / 2} / d$, and $A, B$ and $C$ are given by

$$
A=\frac{\Delta p(1-v)}{\mu}, B=\varepsilon^{3}-\frac{\varepsilon^{6}(1+v)}{2(7-5 v)}, C=\frac{15(2-v) \varepsilon^{6}}{4(7-5 v)}
$$

Here, $\epsilon=a / d$, and $\nu$ is Poisson's ratio.

The strain field produced by contraction of the sphere can be transformed into a stress field by Hooke's law, and the stress field can then be evaluated using a dimensionless factor $F$ based on the Coulomb failure criterion (e.g., Jaeger and Cook, 1969; Segall and Pollard, 1980), as follows.

$$
F \equiv \frac{1}{c_{0}}\left[\sigma_{1}-\sigma_{3} \tan ^{2}\left(45^{\circ}+\frac{\varphi}{2}\right)\right]
$$

where $\sigma_{1}$ and $\sigma_{3}$ are the maximum and minimum principal stresses, and $c_{0}$ and $\phi$ are the compressive strength and angle of internal friction. As the crust is assumed to react as an elastic-perfectly plastic material, the area of $F \geq 1$ corresponds to the plastic and/or rupture area. The relationships between radius and depth and between depth and radial deformation of the chamber were estimated for the stage of formation of the first plastic area on the surface. In addition, the relationship between caldera geometry (type) and the depth of the magma chamber was discussed by comparing the radius of the contracted chamber with the radius of the initial caldera on the surface.

The simulations were conducted assuming basaltic crust with the following elastic constants: Poisson ratio of 0.25 $(\lambda=\mu)$, Young modulus of $40 \mathrm{GPa}$, and compression strength $\left(c_{0}\right)$ of $160 \mathrm{MPa}$ (e.g., Gudmundsson, 1988; Yamaji, 2000). The angle of internal friction was assumed to be $30^{\circ}$ considering the observed angle for rock near the surface (e.g., Yamaji, 2000).

\section{Results and Discussion}

Figure 2(a) shows the relationships between the depth and contraction of the magma chamber at the stage of formation of the first plastic area on the surface, and Figure 2(b) shows the relationship between the depth and horizontal distance from the top of the magma chamber to the plastic area. The depth $(d)$, contraction $(\Delta a)$ and horizontal distance $(r)$ shown in Fig. 2 are normalized by the radius $(a)$ of the magma chamber before contraction. From these figures, it can be determined that the difference between the point source model and the finite sphere model does not seriously influence the results, whereas differences in the analytical dimensions have a marked effect. This result indicates that the relationships between $\Delta a / a$ and $d / a$ and between $r / a$ and $d / a$ can be reasonably estimated using the point source model.

The regression curves explaining the $\Delta a / a-d / a$ and $r / a-$ $d / a$ relations for the point source model were obtained by a least-squares fit of the data. The $\Delta a / a-d / a$ relation is given by

$$
\left(\frac{\Delta a}{a}\right)=\sum_{n=0}^{5} \kappa_{n}\left(\frac{d}{a}\right)^{n}
$$

where $\kappa_{0}=-2.13 \times 10^{-1}, \kappa_{1}=4.02 \times 10^{-1}, \kappa_{2}=-2.70 \times$ $10^{-1}, \kappa_{3}=8.30 \times 10^{-2}, \kappa_{4}=-1.16 \times 10^{-2}$ and $\kappa_{5}=$ $6.18 \times 10^{-4}$. Due to the selection of the lowest degree that explains the data, the physical meaning of each coefficient is unknown at present. However, it can be expected that Eq. (1) is free of serious error considering that the necessary collapse $(\Delta a / a)$ for caldera formation is described by a polynomial of $d / a$. 

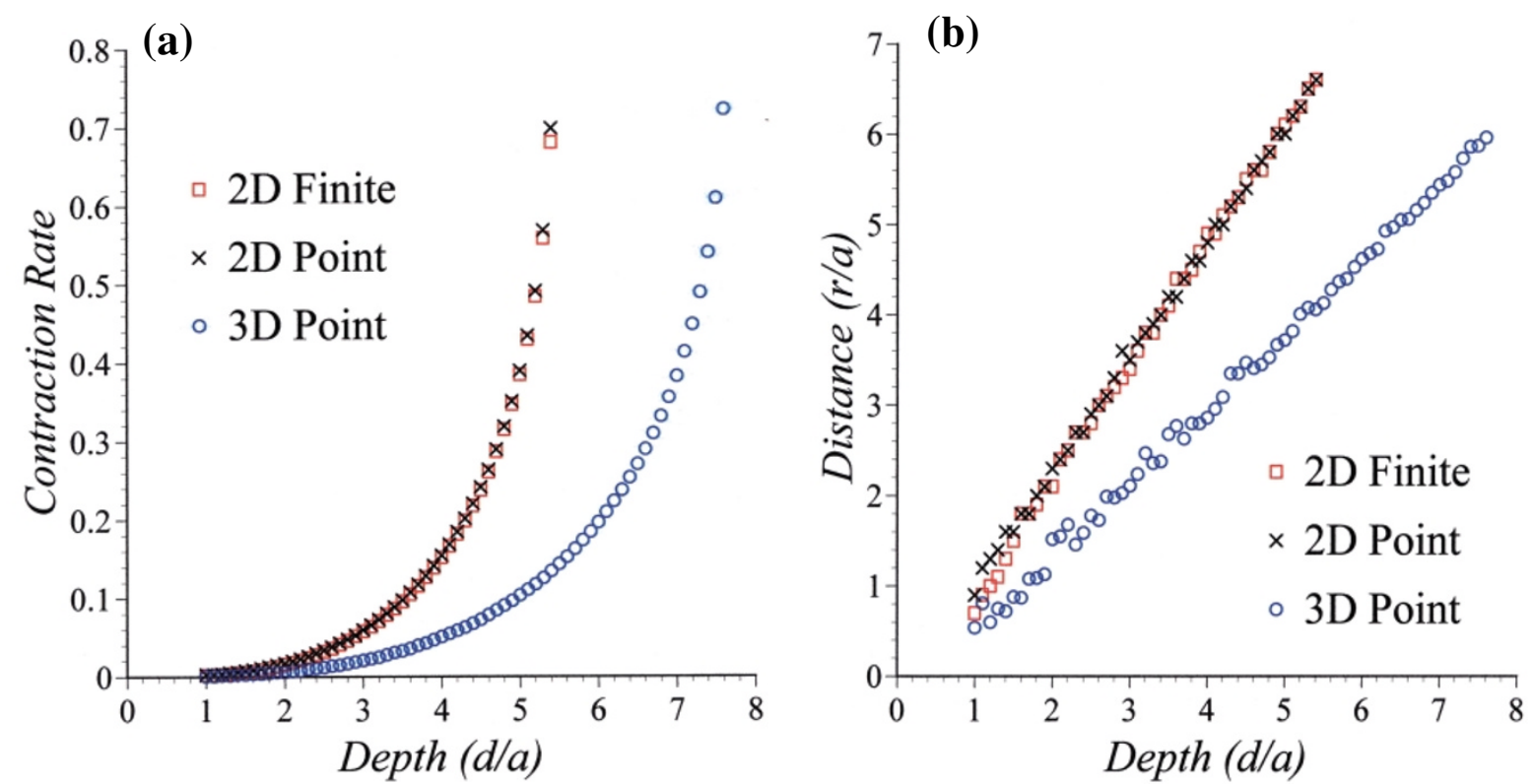

Fig. 2. (a) The $\Delta a / a-d / a$ relation between depth and contraction of the magma chamber. The necessary contraction for the formation of a caldera is described by a polynomial of $d / a$. (b) The $r / a-d / a$ relation between depth and horizontal distance from the top of the magma chamber to the plastic area. The radius of the initial caldera depends on the depth of the magma chamber. Differences in analytic dimensions have a substantial effect on the results, whereas the size of the sphere does not.

The $r / a-d / a$ relation is given by

$$
\left(\frac{r}{a}\right)=\alpha\left(\frac{d}{a}\right)
$$

where $\alpha=0.82$. The proportionality coefficient indicates that the angle ( $\delta$; Fig. 1$)$ between the centre of the magma chamber and the initial caldera boundary is always ca. $39.4^{\circ}$. It is well known that the width of the magma chamber controls the location of the ring faults (e.g., Gudmundsson et al., 1997; Roche et al., 2000). The relation shown in here indicates that not only the width but also the depth of the chamber is important factor which controls the radius of the initial caldera.

The relationship between the depth of the magma chamber and the angle $\theta$ (Fig. 3), defined as the angle between the initial caldera boundary at the surface and a perpendicular line drawn from the edge of the contracted chamber to the surface, was found to depend on $d / a$, with a negative value at $d / a \leq 1.7$ and a positive value at $d / a>1.7$.

A negative angle $(\theta \leq 0)$ indicates that the radius of the initial caldera is smaller than the radius of the magma chamber after contraction, resulting in the formation of a piston caldera. Three end-member collapse geometries have been proposed for piston calderas (e.g., Roche et al., 2000); inward-dipping faults, vertical- or outward-dipping ring faults, and a trapdoor geometry. In most cases, the dip angles of the ring faults are very steep, typically $70-80^{\circ}$ as reported in field studies (e.g., Yoshida, 1984; Rymer et al., 1998). In the case of $\theta \leq 0$, it is thus expected that a piston caldera with outward-dipping or vertical ring faults will be formed.

A result of $\theta>0$, on the other hand, indicates that the radius of the initial caldera is larger than the radius of the chamber after contraction, resulting in the formation of a

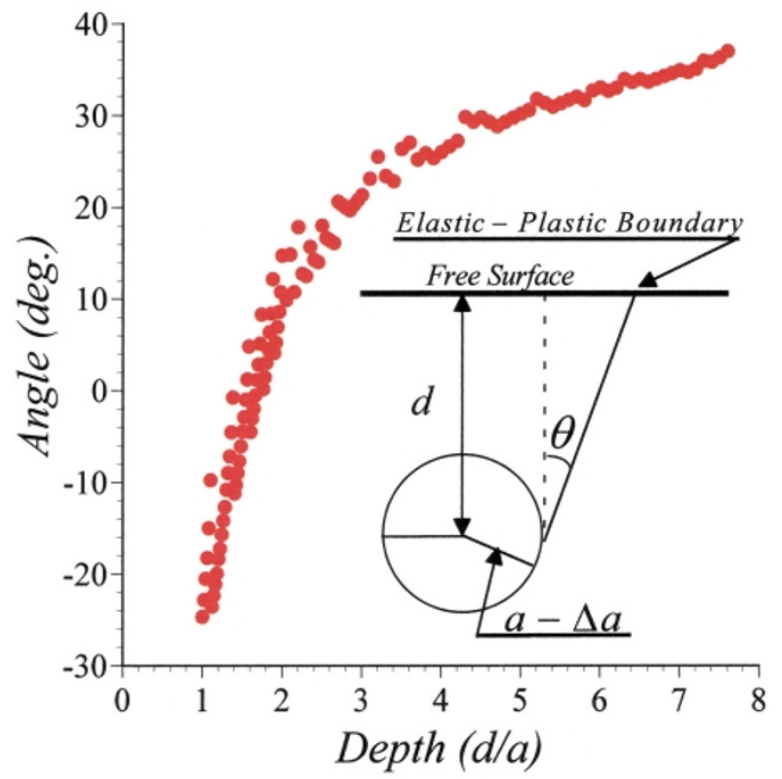

Fig. 3. Relationship between the depth of the magma chamber and the angle $\theta$. The sign and magnitude of $\theta$ indicate the caldera type: $\theta \leq 0$ indicates the formation of a piston caldera with outward-dipping or vertical ring faults, and $\theta>0$ indicates the formation of a piston or funnel caldera with inward-dipping ring faults.

funnel caldera or piston caldera with inward-dipping ring faults.

It is difficult to compare quantitatively our results with the findings pointed out by Roche et al. (2000) and Lipman (1997), because the depth and size of the magma chamber for deciding caldera type are not shown in their discussions. However, despite potential differences in the medium and model assumed here, the present results support their find- 
ings. Namely, the funnel and piston calderas have the same collapse mechanism and/or physical processes, and the geometry (type) of the caldera will be controlled by the depth of the magma chamber.

The heterogeneity of elastic constants and the effects of the regional stress field were not considered in the present model. As pointed out by many researchers (e.g., Nakamura, 1977; Gudmundsson, 2002, 2003; Kusumoto and Takemura, 2003), these are important factors in caldera formation and can be expected to influence the final geometry of the caldera. Further research through field studies, laboratory experiments and numerical simulations are therefore necessary to clarify the effect of these factors on caldera formation.

\section{Conclusion}

Quantitative relationships between caldera geometry and the depth of the magma chamber were obtained by evaluation of the stress field using the contraction of a point source in an elastic medium to model the collapse of the magma chamber. It was found that the necessary contraction for the formation of a caldera is described by a fifth-power polynomial expression of the depth of the magma chamber, and that the angle between the centre of the magma chamber and the initial caldera boundary is always ca. $39.4^{\circ}$. The depth of the magma chamber was also found to determine the radius and geometry of the caldera, and thus to govern whether a piston- or funnel-type caldera is eventually formed.

Acknowledgments. We are thankful to Professor A. Gudmundsson of University of Gottingen and Professor R. Funiciello of Universita Degli Studi Roma TRE for their careful reviews of the paper and helpful comments on the manuscript. This work was done as a part of the Unzen Scientific Drilling Project of the Ministry of Education, Cultures, Sports, Science and Technology of Japan and was also partly supported by a Grant in Aid for Scientific Research from the Ministry of Education, Cultures, Sports, Science and Technology of Japan (No. 14208045 and 17740292).

\section{References}

Acocella, V., F. Cifelli and R. Funiciello, Analogue models of collapse calderas and resurgent domes, J. Volcanol. Geotherm. Res., 104, 81-96, 2000 .
Gudmundsson, A., Effect of tensile stress concentration around magma chambers on intrusion and extrusion frequencies, J. Volcanol. Geotherm. Res., 35, 179-194, 1988.

Gudmundsson, A., Formation and development of normal-fault calderas and the initiation of large explosive eruptions, Bull. Volcanol., 60, 160 $170,1998$.

Gudmundsson, A., Emplacement and arrest of sheets and dykes in central volcanoes, J. Volcanol. Geotherm. Res., 116, 279-298, 2002.

Gudmundsson, A., Surface stresses associated with arrested dykes in rift zones, Bull. Volcanol., 65, 606-619, 2003.

Gudmundsson, A., J. Marti, and E. Turon, Stress fields generating ring faults in volcanoes, Geophys. Res. Lett., 24, 1559-1562, 1997.

Hagiwara, Y., Theory of Geogravity, Kyoritsu-zensyo, Tokyo, 242 pp., 1978 (in Japanese)

Jaeger, J. C. and N. G. Cook, Fundamentals of Rock Mechanics, Methuen, London, 513 pp., 1969.

Komuro, H., Experiments on cauldron formation: a polygonal cauldron and ring fractures, J. Volcanol. Geotherm. Res., 31, 139-149, 1987.

Kusumoto, S. and K. Takemura, Numerical simulation of caldera formation due to collapse of a magma chamber, Geophys. Res. Lett., 30(24), 2278, doi10.1029/2003GL018380, 2003.

Lipman, P. W., Subsidence of ash-flow calderas: relation to caldera size and magma-chamber geometry, Bull. Volcanol., 59, 198-218, 1997.

Marti, J., G. J. Ablay, L. T. Redshaw, and R. S. J. Sparks, Experimental studies of collapse calderas, J. Geol. Soc. London, 151, 919-929, 1994.

McTigue, D. F., Elastic stress and deformation near a finite spherical magma body: resolution of the point source paradox, J. Geophys. Res., 92, 12931-12940, 1987.

Mogi, K., Relations between eruptions of various volcanoes and the deformation of the ground surface around them, Bull. Earthquake Res. Inst., 36, 99-134, 1958.

Nakamura, K., Volcanoes as possible indicators of tectonic stress orientation-principle and proposal, J. Volocanol. Geotherm. Res., 2, 1-16, 1977.

Roche, O., T. H. Druitt, and O. Merle, Experimental study of caldera formation, J. Geophys. Res., 105, 395-416, 2000.

Rymer, H., B. van Vries, J. Stix, and G. Williams-Jones, Pit crater structure and processes governing persistent activity at Masaya volcano, Nicaragua, Bull. Volcanol., 59, 345-355, 1998.

Segall. P. and D. D. Pollard, Mechanics of Discontinuous Faults, J. Geophys. Res., 85, 4337-4350, 1980.

Tsuchida, E. and I. Nakahara, Stresses in a semi-infinite body subjected to uniform pressure on the surface of a cavity and the plane boundary, Bull. JSME, 15, 1-12, 1972.

Yamaji, A., Introduction to Theoretical Tectonics, Asakura Syoten, Tokyo, 287 pp., 2000 (in Japanese).

Yoshida, T., Tertiary Ishizuki cauldron, southwestern Japan arc formation by ring fracture subsidence, J. Geophys. Res., 89, 8502-8510, 1984.

S. Kusumoto (e-mail: kusu@scc.u-tokai.ac.jp) and K. Takemura 